\title{
Harmonic radiation of a relativistic nonlinear inverse Compton scattering using two laser wavelengths
}

\author{
Y. Sakai, O. Williams, G. Andonian, A. Fukasawa, E. Hemsing, A. Marinelli, \\ S. Barber, F. H. O'Shea, and J. B. Rosenzweig \\ UCLA Department of Physics and Astronomy, 405 Hilgard Avenue, Los Angeles, California 90095, USA
}

(Received 29 September 2011; published 28 December 2011)

\begin{abstract}
The use of two different wavelength lasers in the nonlinear regime of the inverse Compton scattering interaction is proposed in order to provide a new strategy for controlling scattered photon energy distributions in the $\mathrm{x}$-ray to $\gamma$-ray spectral region. In this nonlinear interaction, the component of the relativistic electron's trajectory driven by a longer-wavelength laser with the normalized vector potential $a_{\mathrm{L}} \sim 1$ is a large oscillatory figure-8; in the proposed scenario a rapid small-amplitude oscillation induced by a shorter-wavelength laser is superimposed upon this figure-8. Thus, the electron's momentum is mainly supplied from longer-wavelength laser, while the high-frequency part of the acceleration is given by shorter-wavelength laser. In this way, the harmonics radiated at high frequency from the oscillating electron can be strongly modified by the nonlinear motion initiated by the low frequency, large $a_{\mathrm{L}}$ laser resulting in the generation of the harmonics with the photon energy of $4 \gamma^{2} \hbar\left(\omega_{\mathrm{L}, \text { short }}+n \omega_{\mathrm{L}, \text { long }}\right)$. In this paper, the electron's kinetics in the two-wavelength laser field and the concomitant emitted radiation spectrum are examined, with numerical illustrations based on a classical Lienard-Wiechert potential formalism provided.
\end{abstract}

\section{INTRODUCTION}

Inverse Compton scattering (ICS) is an attractive scheme for producing narrow-band $\mathrm{x}$ rays having energies in the $\mathrm{keV}$ range or above, while at the same time using relatively lowcompared to an x-ray free-electron laser, e.g.-electron beam energy. The Doppler shifting of an incident laser scattered by a relativistic beam electron in the Thomson limit produces a backscattered $\mathrm{x}$ ray in the energy of [1]

$$
\hbar \omega_{\mathrm{x}-\text { ray }}=\frac{4 \gamma^{2} \hbar \omega_{\mathrm{L}}}{1+a_{\mathrm{L}}^{2} / 2+\gamma^{2} \theta^{2}},
$$

where $\gamma$ is the electron's Lorentz relativistic factor, $a_{\mathrm{L}}$ is the normalized vector potential, and $\theta$ is the observation angle relative to the incident electron direction. Thus, the backscattered photon energy is directly proportional to the incident laser energy $\hbar \omega_{\mathrm{L}}$ and to the relativistic Doppler factor $4 \gamma^{2}$. Recently, production of subpicosecond, high flux $\mathrm{keV} \mathrm{x}$ rays using the ICS technique [2-5], as well as their use in various applications, have been proposed and demonstrated [6-8]. These applications have included phase contrast imaging [9] and single shot diffraction from a crystal [10]. The demonstrated scattered photon number per ICS interaction enabling these applications is $\sim 10^{7}-10^{8}$.

To increase the energy efficiency of scattered photon production, the excitation of harmonic $\mathrm{x}$ rays by nonlinear ICS has been investigated theoretically in recent decades

Published by the American Physical Society under the terms of the Creative Commons Attribution 3.0 License. Further distribution of this work must maintain attribution to the author(s) and the published article's title, journal citation, and DOI.
[11-13]; and radiation at the 2nd harmonic has been observed by using an intense $\mathrm{CO}_{2}$ laser with $a_{\mathrm{L}} \sim 1$ [14]. As $a_{\mathrm{L}}$, as mentioned above, is defined as the normalized vector potential of the laser wave, it is equivalent to the momentum associated with the electron's transverse oscillation in the laser wave field $E_{\mathrm{L}}$, divided by $m_{e} c$ [11],

$$
a_{\mathrm{L}}=\frac{e E_{\mathrm{L}}\left(\lambda_{\mathrm{L}} / 2 \pi\right)}{m_{e} c^{2}}
$$

where $m_{e}$ and $e$ are the electron mass and charge, respectively, $\lambda_{\mathrm{L}}$ is the wavelength of the laser, and $c$ is the velocity of the light. As one can see from Eq. (2), in order to induce strong transverse motion, it is advantageous to use a longwavelength laser to increase the acceleration time in the laser cycle. However, use of a long wavelength not only lowers the incident photon energy itself, but also large $a_{\mathrm{L}}$ lowers the scattered $x$-ray energy via redirecting the beam electrons away from the nominal propagation direction $z$ (Fig. 1). Here, therefore, we examine the possibility of using a small-amplitude rapid oscillation induced by a

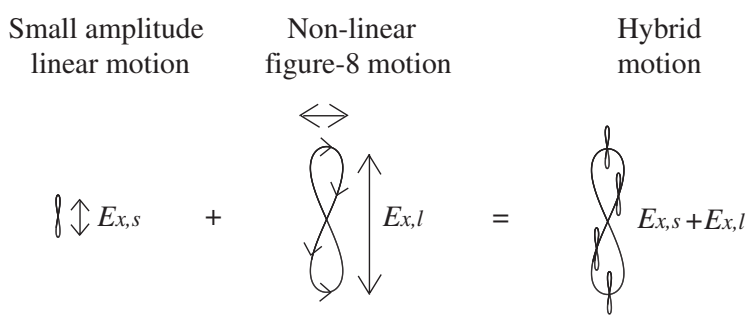

FIG. 1. Schematic diagram of the nonlinear (figure-8) motion induced by a shorter- and a longer-wavelength laser. $E_{s}, E_{l}$ : Electric field of shorter- and longer-wavelength lasers, respectively. 
short-wavelength laser in the presence of the large orbital figure- 8 motion induced by a long-wavelength laser. This will permit us to take advantage of aspects of the nonlinear motion, such as harmonic production, while obtaining higher energy x rays, with the energy scale set by a shortwavelength laser that is not in itself capable of driving nonlinear motion. This technique also provides excellent hard $\mathrm{x}$-ray tunability, by simply changing the e-beam energy using the recently emerging intense $\mathrm{CO}_{2}$ laser with $E_{\mathrm{L}} \sim \mathrm{TV} / \mathrm{m}[14,15]$.

The physics contained in the superimposed two-laser scheme can be related to that of scenarios in the freeelectron-laser context [16] that employ two-frequency wigglers [17] and biharmonic undulators [18,19], that are utilized for better control of electrodynamics and emission spectrum. In the two-laser ICS case, the amplitude of the electron's oscillatory momentum is mainly supplied from longer-wavelength laser. The high-frequency acceleration which has as its objective the production of high energy photons is given by the shorter-wavelength laser, thus generation of harmonic $\mathrm{x}$ rays with photon energy of $4 \gamma^{2} \hbar\left(\omega_{\mathrm{L} \text {,short }}+n \omega_{\mathrm{L}, \mathrm{long}}\right)$ could be anticipated.

\section{PHYSICAL ASPECTS OF ELECTRON MOTION AND RADIATION EMISSION}

To analyze this scheme, we need to understand the electron motion in the dual laser field, and then proceed through a Lienard-Wiechert (LW) treatment to deduce the characteristics of the emitted electromagnetic radiation. Let us consider a highly relativistic electron with an initial energy of $\mathcal{E}_{0}$ and position $(x, z)=(0,0)$ counterpropagating along the $z$ axis in two linearly $x$-polarized sinusoidal wave fields. The $x$ and $z$ components of the momentum $\boldsymbol{p}$ in the electron frame are approximated as [20]

$$
p_{x} \cong e A_{x} / c, \quad p_{z} \cong \mathcal{E}_{0} / c,
$$

where the combined vector potential of two lasers is $A_{x}=A_{x, s}+A_{x, l}=A_{0, s} \cos \omega_{s} t+A_{0, l} \cos \omega_{l} t$, having angular frequencies

$$
\begin{aligned}
& \omega_{s}=\omega_{\mathrm{L}, s}\left(c+v_{z}\right) / c \cong 2 \omega_{\mathrm{L}, s} \\
& \omega_{l}=\omega_{\mathrm{L}, l}\left(c+v_{z}\right) / c \cong 2 \omega_{\mathrm{L}, l},
\end{aligned}
$$

written for mathematical convenience in the Galilean frame moving with the Compton interacting electron. Here, the subscripts $s$ and $l$ denote short- and long-wavelength laser, respectively. In Eq. (3), the approximation is assumed that the interacting electron is highly relativistic, and the velocity components of an electron with propagation angle $\theta$ with respect to the $z$ axis are expressed as

$$
\begin{aligned}
& v_{x}=c \sin \theta \approx c \frac{p_{x}}{p_{z}} \cong c \frac{e\left(A_{x, s}+A_{x, l}\right)}{\mathcal{E}_{0}} \\
& v_{z}=c \cos \theta \approx c \sqrt{1-\left(\frac{p_{x}}{p_{z}}\right)^{2}} \cong c\left\{1-\frac{1}{2}\left(\frac{e A_{x}}{\mathcal{E}_{0}}\right)^{2}\right\} .
\end{aligned}
$$

Hence, time integration of $v_{z}$ in $t_{l}$ on the time scale of the longer-wavelength laser period $T_{l}$ gives the longitudinal position $z$ of the electron as

$$
\begin{aligned}
z & \approx \int c\left\{1-\frac{1}{2}\left(\frac{e A_{0, s} \cos \omega_{s} t+e A_{0, l} \cos \omega_{l} t}{\mathcal{E}_{0}}\right)^{2}\right\} d t \\
& =\int c\left\{-\frac{1}{2}\left(\frac{e}{\mathcal{E}_{0}}\right)^{2}\left(\begin{array}{c}
A_{0, s}{ }^{2} \cos ^{2} \omega_{s} t+A_{0, l}{ }^{2} \cos ^{2} \omega_{l} t \\
+2 A_{0, s} \cos \omega_{s} t A_{0, l} \cos \omega_{l} t
\end{array}\right)\right\} d t \\
& \rightarrow \int_{0}^{t_{l}} c\left\{1-\frac{1}{2}\left(\frac{e}{\mathcal{E}_{0}}\right)^{2} A_{0, l}{ }^{2} \cos ^{2} \omega_{l} t\right\} d t \\
& \cong c\left\{t_{1}\left(1-C_{\text {shift }}\right)-\frac{1}{2 \pi} \frac{T_{l}}{2} C_{\text {shift }} \sin 2 \omega_{l} t_{l}\right\} \lesssim c t_{l},
\end{aligned}
$$

where $C_{\text {shift }}=1-\cos \theta$, with propagation angle $\theta=$ $\tan ^{-1}\left[e\left(A_{0, l} / \sqrt{2}\right) / \mathcal{E}_{0}\right]$. In the middle of Eq. (6), the $\cos ^{2} \omega_{s} t$ and $\cos \omega_{s} t \cos \omega_{l} t$ terms are canceled because no net spatial displacement is obtained from the higher frequency oscillation field when integration is performed with respect to the longer time scale with $A_{0, s}{ }^{2} \ll A_{0, l}{ }^{2}$. Thus, the amplitude of the electron's oscillatory momentum is mainly supplied from longer-wavelength laser.

We now examine the far-field Lienard-Wiechert potential along the observation direction $\mathbf{n}$ [21], with the simplification of setting the observing direction along the $z$ axis and concentrating on $x$-polarized component,

$$
\begin{aligned}
\boldsymbol{E}_{\mathrm{LW}} & =\frac{m_{e}}{e} \frac{r_{e}}{R} \frac{\mathbf{n} \times\{(\mathbf{n}-\boldsymbol{v} / c) \times \boldsymbol{w}\}}{(1-\mathbf{n} \cdot \boldsymbol{v} / c)^{3}} \\
\rightarrow-\frac{m_{e}}{e} & \frac{r_{e}}{R_{0}} \frac{\overbrace{w_{x}\left(1-\frac{\boldsymbol{v}_{z}}{c}\right)+\frac{w_{z} v_{x}}{c}}^{\text {contains high frequency factor }}}{\underbrace{\left(1-\frac{\boldsymbol{v}_{z}}{c}\right)^{3}}_{\text {with cycle of figure-8 motion }}} .
\end{aligned}
$$

The Fourier transform of Eq. (7) yields the distribution of the differential radiation intensity spectrum (in solid angle $\Omega$ and angular frequency $\omega$ ) [22], having two frequency components,

$$
\begin{aligned}
\frac{d^{2} I}{d \omega d \Omega} & =\frac{e^{2} \omega^{2}}{4 \pi^{2} c^{3}}\left|\int_{-\infty}^{\infty} \mathbf{n} \times[\mathbf{n} \times \boldsymbol{v}] e^{i \omega(t-\mathbf{n} \cdot \boldsymbol{r} / \boldsymbol{c})}\right| \\
& \rightarrow \frac{e^{2} \omega_{\mathrm{x}-\mathrm{ray}}}{4 \pi^{2} c^{3}}\left|\int_{-\infty}^{\infty} v_{x} e^{i \omega_{\mathrm{x}-\mathrm{ray}}[t-(z / c)]} d t\right|^{2} \\
& \equiv \frac{1}{c}\left|\frac{1}{\lambda_{\mathrm{x}-\text { ray }}}\left\{j_{\omega_{\mathrm{x}-\mathrm{ray}}, s}+j_{\omega_{\mathrm{x}-\mathrm{ray}}, l}\right\}\right|^{2} .
\end{aligned}
$$

Here, in Eqs. (7) and (8), $r_{e}$ is the classical electron radius, $R$ is the distance between a source electron and an observation position, $\boldsymbol{w}$ is the acceleration of the electron, and the $j_{\omega}$ are the Fourier amplitudes of the source currents associated with the biperiodic electric field of the lasers. Hence, the production of harmonic $\mathrm{x}$ rays using two laser wavelengths can be anticipated by the expansion of the oscillatory exponent in Eq. (8) as 


$$
\begin{aligned}
v_{x} \exp & \left\{i \omega_{\text {x-ray }}(t-z / c)\right\} \\
= & \frac{c e}{\mathcal{E}_{0}} \exp \left(i C_{\text {shift }} \omega_{\text {x-ray }} t\right)\left(A_{0, s} \cos \omega_{s} t+A_{0, l} \cos \omega_{l} t\right) \\
& \times \sum_{n=-\infty}^{\infty} J_{n}\left(\frac{C_{\text {shift }} \omega_{\text {x-ray }}}{2 \omega_{l}}\right) \exp \left(i n 2 \omega_{l} t\right),
\end{aligned}
$$

using the identity $\exp (i b \sin \sigma)=\sum_{n=-\infty}^{\infty} J_{n}(b) \exp (\operatorname{in} \sigma)$ with $J_{n}$ the Bessel function of the first kind. Therefore, the components of the transverse momentum oscillation of the electrons (proportional to $A_{x, s} \cos \omega_{s} t+A_{x, l} \cos \omega_{l} t$ ) having angular frequency $n 2 \omega_{l}$ imply generation of harmonics in the emitted photon energy of $\hbar\left(\omega_{s}+n 2 \omega_{l}\right)$ with a Doppler boost factor of $\exp \left(i C_{\text {shift }} \omega_{\text {x-ray }}\right)$. As a result, the observed x-ray energy on the $z$ axis associated with $j_{\omega, \mathrm{x}-\mathrm{ray}, s}$ and $j_{\omega, \mathrm{x} \text {-ray }, l}$ are written as

$$
\begin{aligned}
& \hbar \omega_{\mathrm{x}-\mathrm{ray}, s} \approx 2 \hbar\left(\omega_{\mathrm{L}, s}+2 n \omega_{\mathrm{L}, l}\right) / C_{\text {shift }} \\
& \hbar \omega_{\mathrm{x}-\mathrm{ray}, l} \approx 2 \hbar\left(\omega_{\mathrm{L}, l}+2 n \omega_{\mathrm{L}, l}\right) / C_{\text {shift }} .
\end{aligned}
$$

The $\cos ^{2} \omega_{s} t$ and $\cos \omega_{s} t \cos \omega_{l} t$ terms neglected in Eq. (6) produce low-intensity harmonics due to the small-amplitude figure- 8 motion induced by the shorterwavelength laser by itself, as well as by the complicated combined nonlinear motion induced by the two lasers. The off-axis high-frequency spectrum displays even harmonics of the energies $\hbar \omega_{s}+n \hbar \omega_{l}$, since the denominator in Eq. (7) also becomes a function of transverse velocity $v_{x, l}$.

It may be of importance to note that, although the spectrum on the $z$ axis using only one laser wavelength creates photons having energy $2 \hbar \omega_{l}(1 / 2+n)$, if the angular frequency of $\omega_{s}$ is low enough $\omega_{s} \ll \omega_{l}$, which is opposite case of the objective condition, with amplitude of $a_{\mathrm{L}, s} \ll$ $a_{\mathrm{L}, l}$, the energy of fundamental radiated wavelength may be negligible. Thus, the emitted photon energy on the $z$ axis in the two relevant limits is $\hbar\left(\omega_{s}+n 2 \omega_{l}\right) \rightarrow^{\omega_{l} / \omega_{s} \rightarrow 0} \hbar \omega_{s}$ and $\hbar\left(\omega_{s}+n 2 \omega_{l}\right) \rightarrow^{\omega_{s} / \omega_{l} \rightarrow 0} 2 \hbar \omega_{l} n$.

The field $E_{\mathrm{LW}, x}$ in Eq. (7) behaves in a similar fashion to a $\delta$ function, due to the terms higher order in $\gamma$, in that it has maxima when longitudinal velocity $v_{z}$ approaches $c$. In this scenario, $p_{z}$ may be considered mainly a function of the vector potential of the large wavelength laser. Thus, it is possible to enhance the high-frequency factor in the numerator only where $v_{z}$ approaches $c$, where transverse momentum nearly vanishes, $p_{x} \rightarrow 0$ (at the top and the bottom of the figure- 8 trajectory shown in Fig. 1). In Eqs. (8) and (9), an x-ray energy around the angular frequency of $\omega_{s}$ is obtained from high frequency $\cos \omega_{s} t$ term multiplied by the Bessel term that is weighted by low frequency figure- 8 rotation $\exp \left(i n 2 \omega_{l} t\right)$. Since, the Bessel term is normalized, the summation of these terms is not significantly changed. Indeed, the total x-ray energy $\Delta \varepsilon_{\text {rad }}$ along the figure- 8 path is approximated by utilizing the total radiation energy written in terms of the external electric and magnetic field [21] as

$$
\begin{aligned}
\Delta \varepsilon_{\mathrm{rad}} & =\frac{2}{3} c r_{e}^{2} \int \gamma^{2}\left\{\left(\boldsymbol{E}+\frac{\boldsymbol{v}}{c} \times \boldsymbol{H}\right)^{2}-\left(\boldsymbol{E} \cdot \frac{\boldsymbol{v}}{c}\right)^{2}\right\} d t \\
& \rightarrow \int \frac{1}{r_{e} / 2 c} \frac{4 \pi r_{e}^{3}}{3} 2 \gamma^{2} \frac{1}{8 \pi}\left\{\begin{array}{c}
E_{\mathrm{L}, x}{ }^{2}+\beta^{2} H_{\mathrm{L}, y}{ }^{2} \\
\left.-2 \frac{v_{z, l}+\Delta v_{z, s}}{c} E_{\mathrm{L}, x} H_{\mathrm{L}, y}\right\} \\
-E_{\mathrm{L}, x}{ }^{2}\left(\frac{v_{x, l}+\Delta v_{x, s}}{c}\right)^{2}
\end{array}\right\} \\
& \approx c \sigma_{\mathrm{T}} 2 \gamma_{0}{ }^{2} \frac{\left(E_{\mathrm{L}, \mathrm{rms}, s}+E_{\mathrm{L}, \mathrm{rms}, l}\right)^{2}}{4 \pi}\left\{2-\left(\frac{a_{\mathrm{L}, \mathrm{rms}, l}}{\gamma_{0}}\right)^{2}\right\} \Delta t_{\mathrm{int}},
\end{aligned}
$$

Thus, although, in addition to the interference term of the laser field itself, some higher-order corrections due to treating the Lorentz factor as a constant are missing, the total emitted electromagnetic energy along the figure- 8 trajectory, within a $1 / \gamma$ angular cone, is approximately unchanged by the addition of the small $a_{\mathrm{L}}$ field, as long as $v_{x, l} \gg \Delta v_{x, s}$.

\section{NUMERICAL EXAMPLE}

To illustrate the results of our analysis, a single electron orbit is numerically calculated to evaluate the modulated Lienard-Wiechert potential in Eq. (7) and the associated scattered x-ray spectrum generated on an observation screen located in the far field. This calculation proceeds by solving the relativistic equations of motion under the constraint of the energy-momentum relation [21], with the combined laser field assumed to be made up of superimposed linearly $x$-polarized coherent components ignoring the radiation reaction. The relevant equations are thus

$$
\begin{aligned}
\frac{d \boldsymbol{p}}{d t} & =e \boldsymbol{E}+e\left(\frac{\boldsymbol{v}}{c}\right) \times \boldsymbol{H} \quad p_{\mu} p^{\mu}=m_{e}{ }^{2} c^{2} \quad \boldsymbol{v}=\frac{\boldsymbol{p} c}{\mathcal{E}} \\
E_{x} & =E_{0, s} \sin \left(k_{s} z-\omega_{s} t\right)+E_{0, l} \sin \left(k_{l} z-\omega_{l} t\right) .
\end{aligned}
$$

In our example, the driving laser wavelengths are set to $10.6 \mu \mathrm{m}\left(\mathrm{CO}_{2}\right.$ laser), $800 \mathrm{~nm}$ (Ti:sapphire laser). The initial electron energy is taken as $65 \mathrm{MeV}$ [14] as at the BNL ATF. In this case, the backscattered energy of $4 \gamma^{2} \omega_{0}$ is 7.6 and $100 \mathrm{keV}$ for the $\mathrm{CO}_{2}$ laser and the Ti:sapphire laser, respectively. In the numerical calculation, the distance from the screen and the ICS interaction point is set to $1 \mathrm{~m}$.

The calculated $x, z$ component of acceleration $w$, velocity $v$, total radiation energy per unit time $d \Delta \varepsilon_{\text {rad }} / d t$ [Eq. (10)], and $x$-polarized Lienard-Wiechert potential term $E_{\mathrm{LW}, x}\left(t_{\text {electron }}\right)$ in the electron frame with different $a_{\mathrm{L}}$ of $\mathrm{CO}_{2}$ laser are shown in Fig. 2. The bold line in this figure corresponds to that of using only the $\mathrm{CO}_{2}$ laser. The value of $a_{\mathrm{L}}$ associated with the $\mathrm{CO}_{2}$ laser is varied as $0.1,0.5,1.0$ and 1.5 , while that of the Ti:sapphire laser is fixed to $\sim 0.1$.

In Fig. 2, it can be seen that the amplitude of the electron's velocity components, which are approximated 
as $v_{x} \sim c a_{\mathrm{L}, \mathrm{CO} 2} / \gamma$ and $v_{z} \sim c\left\{1-0.5\left(a_{\mathrm{L}, \mathrm{CO} 2} / \gamma\right)^{2}\right\}$, is not significantly affected by effects of the short-wavelength laser, as expected. Further, the doubled-frequency oscillation of $v_{z}$ with respect to that of $v_{x}$ reflects the existence of the large orbital figure- 8 motion induced by the longer-wavelength laser. The acceleration due to the
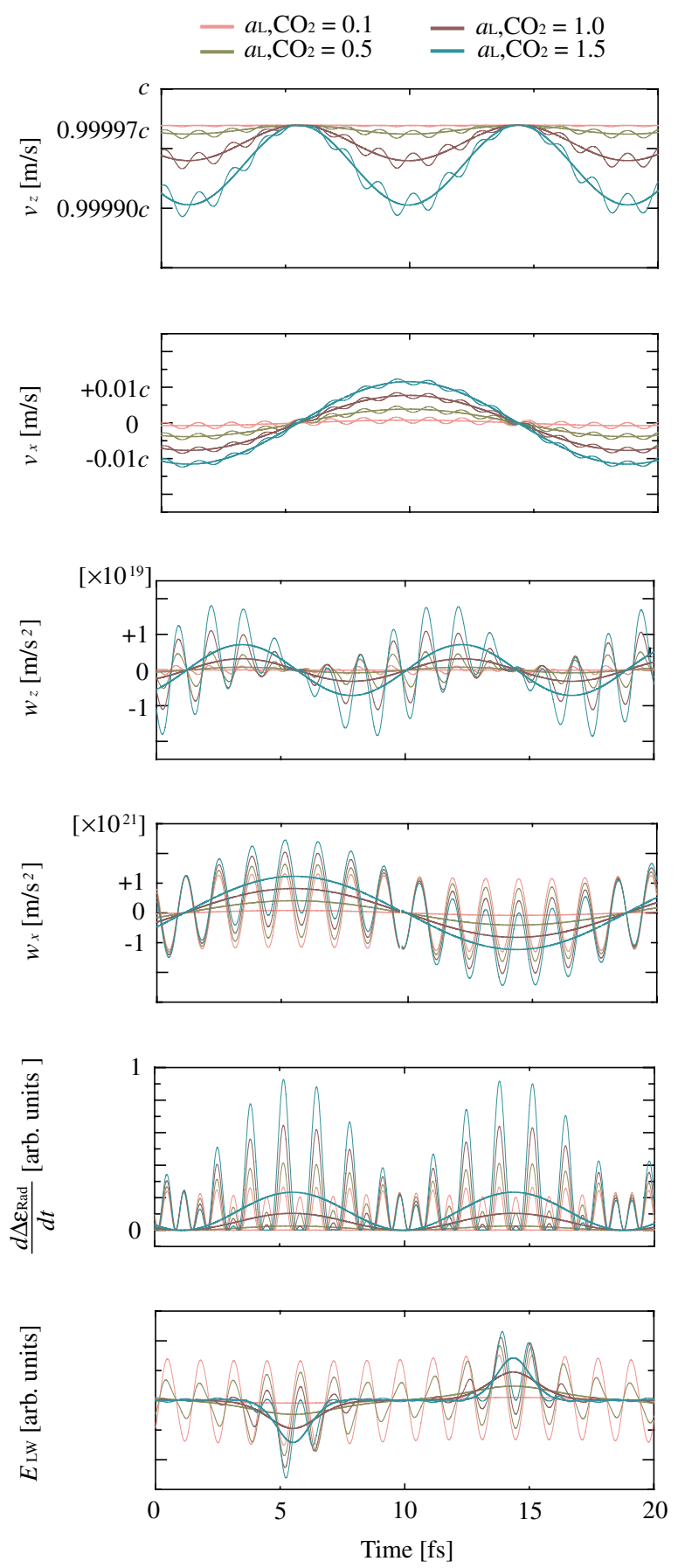

FIG. 2. Numerically calculated $x$ and $z$ components of acceleration $w$ and velocity $v$, total radiation energy per unit time $d \Delta \varepsilon_{\text {rad }} / d t$, and Lienard-Wiechert potential $E_{\mathrm{LW}, x}\left(t_{\text {electron }}\right)$ calculated in the electron frame, varying the $a_{\mathrm{L}}$ of the $\mathrm{CO}_{2}$ laser, with $a_{\mathrm{L}, \mathrm{Tr} \text { :sapphire }}: 0.1$, electron energy of $65 \mathrm{MeV}$. short-wavelength laser field is also modulated by the figure-8 motion. The high-frequency component of the acceleration along the $z$ direction $w_{z}$ is suppressed when $v_{z}$ approaches the $c$ with the maximum kinetic energy, while the $x$ component of acceleration induced by two laser wavelengths are almost linearly superimposed. As a consequence, a strong modulation of the Lienard-Wiechert potential can be anticipated. The instantaneous total radiation energy during the acceleration phase of electron in the figure- 8 motion is increased by almost a factor of 4 due to the interference effect of the superimposed laser. There is, however, a nearly invariant total time integrated radiative energy. Thus, the time squeezing effect and the conservation of energy could be seen.

Figures 3(a) and 3(b) show the calculated $E_{\mathrm{LW}, x}\left(t_{\text {screen }}\right)$ and its on-axis spectrum, $E_{\mathrm{LW}, x}(\omega)=\left|\int_{-\infty}^{\infty} E_{\mathrm{LW}, x}(t) e^{i \omega t} d t\right|$, on the screen, in terms of the screen time $t_{\text {screen }}=t_{\text {electron }}+$ $R\left(t_{\text {electron }}\right) / c$. With an increase of $a_{\mathrm{L}, \mathrm{CO} 2}$ from 0.1 to 0.5 , the wave form of $E_{\mathrm{LW}, x}\left(t_{\text {screen }}\right)$ changes from sinusoidal to triangular. In the cases of $a_{\mathrm{L}, \mathrm{CO} 2}=0.1$ and 0.5 , modulation of $E_{\mathrm{LW}, x}\left(t_{\text {screen }}\right)$ by the high-frequency fields can be observed at any phase of sinusoidal or triangular waves. However, in the case of $a_{\mathrm{L}}=1.0,1.5$, the radiation field induced by the highfrequency laser can be observed only near the spikes. Thus, harmonic radiation as a result of the relativistic figure-8 motion is implied.

In Fig. 3(b), by increasing $a_{\mathrm{L}}$ of the $\mathrm{CO}_{2}$ laser, the redshift of the fundamental radiation is observed to be $\left(a_{\mathrm{L}}, \mathrm{CO} 2\right.$, fundamental energy $\left.[\mathrm{keV}]\right)=(0.1,7.5),(0.5,6.7)$, $(1.0,5.1),(1.5,3.6)$, respectively, in agreement with analytical evaluation using Eq. (1). An increase of $a_{\mathrm{L}, \mathrm{CO} 2}$ to 0.5 generates harmonics in the vicinity of 7.6 and $100 \mathrm{keV}$. Consequently, although the intensity of the each discrete $\mathrm{x}$-ray line at a given harmonic is decreased, the harmonics in the energy of around $150 \mathrm{keV}$ can be anticipated with $a_{\mathrm{L}, \mathrm{CO} 2} \sim 1$, boosted by the harmonics contained in the orbital figure-8 motion.

The angular distribution of the on-axis spectrum is shown in Fig. 3(c), which displays the existence of the odd harmonics in the x-ray energy extending above $100 \mathrm{keV}$. It should be noted that the divergence of the $\mathrm{x}$ rays induced by the short-wavelength laser is smaller than that associated with the longer-wavelength laser.

In the end, the x-ray spectral distribution is controlled both by selection of the ratio of wavelength of two lasers, and by increasing the vector potential of the large wavelength laser, which changes the energy separation of the harmonics, as well as their relative strengths. To illustrate aspects of this control, Figs. 4(a) and 4(b) show the possible modulation of the x-ray spectrum in the energy range around $0.5 \mathrm{MeV}$ in the case of the ICS interaction of a $75 \mathrm{MeV}$ electron beam with a $\mathrm{CO}_{2}$ and $200 \mathrm{~nm}$ (frequencyquadrupled Ti:sapphire, for example) laser. The normalized vector potential of the $\mathrm{CO}_{2}$ and $200 \mathrm{~nm}$ laser field, 
(a)

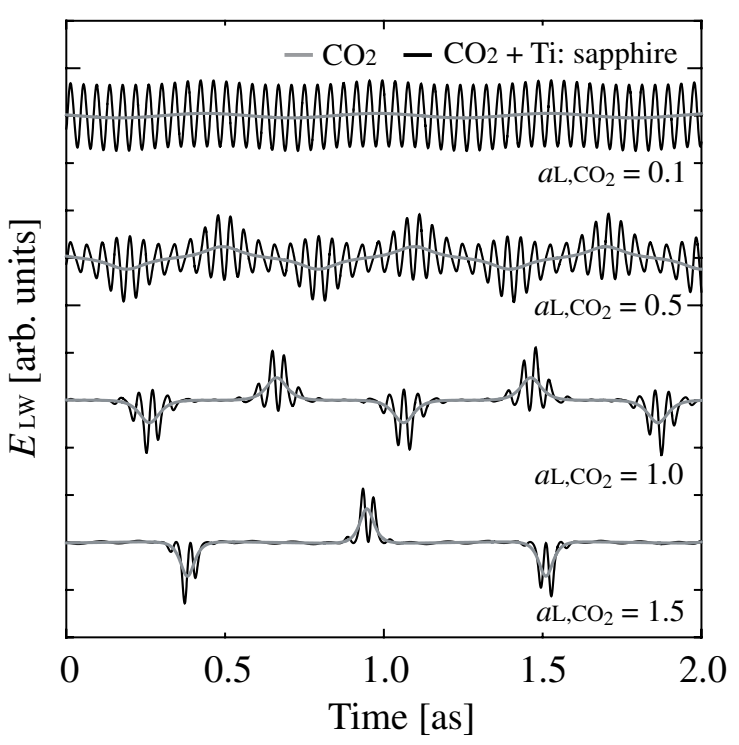

(b)

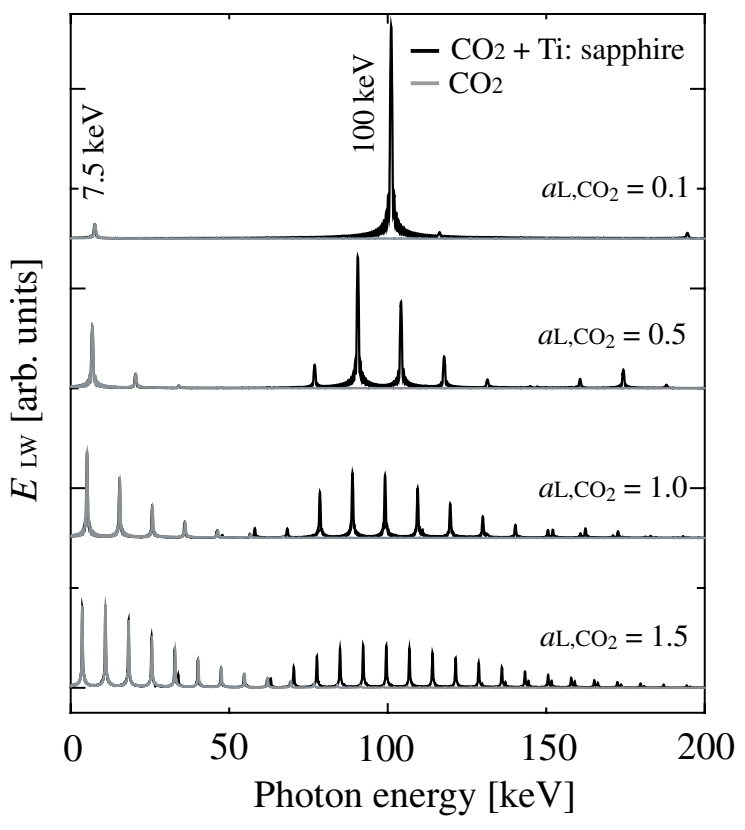

(c)
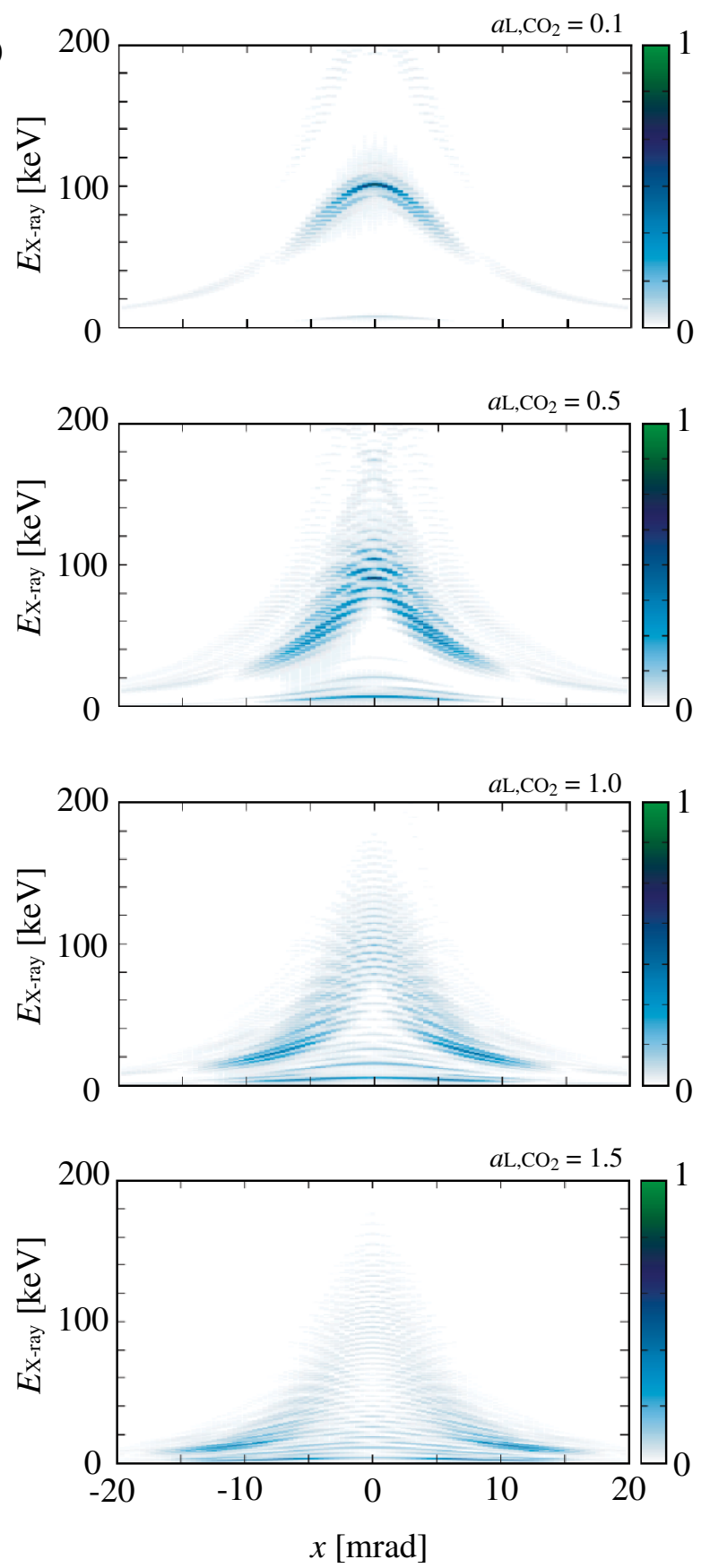

FIG. 3. Numerically calculated Lienard-Wiechert potential $E_{\mathrm{LW}, x}\left(t_{\mathrm{screen}}\right)$ on $(x, y, z)=(0,0,0)(\mathrm{a})$, its spectrum on $(x, y, z)=$ $(0,0,0)(b)$, and its angular spectrum on $(y, z)=(0,0)(\mathrm{c})$, on the screen generated by the interaction of $65 \mathrm{MeV}$ electron and two laser fields with different $a_{\mathrm{L}, \mathrm{CO} 2}$ with $a_{\mathrm{L}, \mathrm{Ti} \text { :sapphire }} \approx 0.1$. Gray line: With only $\mathrm{CO}_{2}$ laser, Black: With $\mathrm{CO}_{2}$ and Ti:sapphire laser. The redshift of the fundamental radiation: $\left(a_{\mathrm{L}, \mathrm{CO} 2}\right.$, fundamental energy $\left.[\mathrm{keV}]\right)=(0.1,7.5),(0.5,6.7),(1.0,5.1),(1.5,3.6)$.

respectively, is set to $a_{\mathrm{L}}=2.0$ and $2.0 / 50=0.04$, and backscattered value is $0.51 \mathrm{MeV}$. By adjusting the ratio of the two laser wavelengths and the electron beam energy, it is possible to match the peak in reaction cross section of atomic, molecular, and even nuclear processes [8], as this example of $\mathrm{MeV}$-class photon generation shows.

In this way, inverse Compton scattering, using the superposition of two different laser fields, and exploiting nonlinear effects, can thus generate multiple energy line electromagnetic radiation beams that would be able to provide unique opportunities in hard photon-based experiments. For example, by angular separation of the ICS lines using diffraction methods, one can generate multiple beams to excite simultaneously different physical systems, or create new pump-probe scenarios. Even without separation of the different energy beams, one may simultaneously excite 
(a)

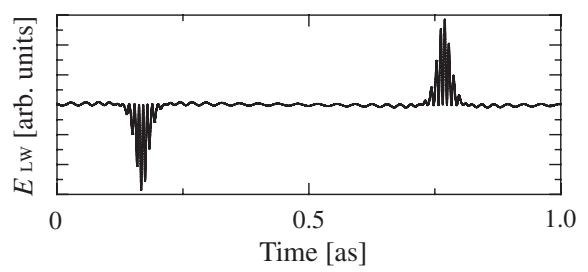

(b)

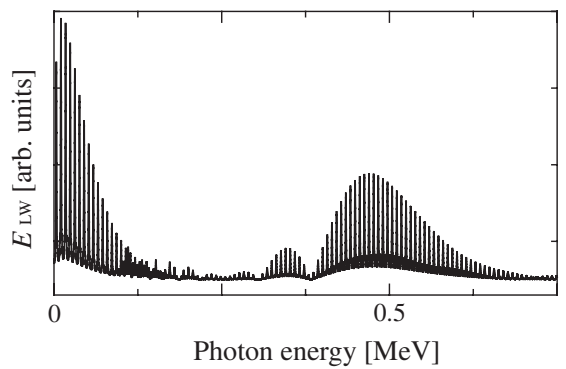

FIG. 4. Numerically calculated $E_{\mathrm{LW}, x}\left(t_{\text {screen }}\right)$ (a), its on-axis spectrum (b), $E_{\mathrm{LW}, x}(\hbar \omega)$ on the screen, that is produced by the ICS interaction of $75 \mathrm{MeV}$ electron beam, $\mathrm{CO}_{2}$ laser, and $200 \mathrm{~nm}$ $(10.6 \mu \mathrm{m} / 50)$ laser. $a_{\mathrm{L}, \mathrm{CO} 2}: 2.0, a_{\mathrm{L}, 200 \mathrm{~nm} \text { laser }}=2.0 / 50 \approx 0.04$ backscattered value: $0.51 \mathrm{MeV}$.

several discrete resonances in a single-species or multiplespecies target system with slightly higher photon energy. We have given a numerical example of the performance of this scheme in the context of the BNL ATF inverse Compton scattering source. The feasibility of performing such an experiment is now under active examination.

\section{SUMMARY}

The possibility of modulating the emitted radiation spectrum by employing two lasers in an ICS interaction in the limit that the longer-wavelength laser induces nonlinear motion has been analyzed. The nonlinear relativistic orbital figure- 8 motion driven by the long-wavelength laser is superimposed upon a small-amplitude electron oscillation induced by a shorter-wavelength laser. Consequently, the harmonic radiation emitted from the high-frequency component of the oscillating electron motion may be strongly modified. Indeed, it is possible to control the $\mathrm{x}$-ray spectral distribution by choice of the ratio of wavelengths of two lasers, and through selective increase of the vector potential of the large wavelength laser. This two-laser scheme would make ICS to be a flexible source of higher energy photon or multiline spectrum photons at $\mathrm{x}$-ray to $\gamma$-ray wavelengths.

\section{ACKNOWLEDGMENTS}

The authors would like to thank all of the members and collaborators in UCLA PBPL and BNL ATF. This work was supported by the U.S. Department of Energy under Contracts No. DE-FG02-07ER46272 and No. DE-FG0392ER40693, the Office of Naval Research under Contract No. ONR N00014-06-1-0925, and the Defense Threat Reduction Agency Contract No. HDTRA1-10-1-0073.

[1] P. Sprangle, A. Ting, E. Esarey, and A. Fisher, J. Appl. Phys. 72, 5032 (1992).

[2] W. P. Leemans, R. W. Schoenlein, P. Volfbeyn, A. H. Chin, T. Glover, P. Balling, M. Zolotorev, K. J. Kim, S. Chattopadhyay, and C. V. Shank, Phys. Rev. Lett. 77, 4182 (1996).

[3] I. V. Pogorelsky et al., Phys. Rev. ST Accel. Beams 3, 090702 (2000).

[4] O. Williams, G. Andonian, M. Babzien, E. Hemsing, K. Kusche, J. Park, I. Pogorelsky, G. Priebe, J. Rosenzweig, and V. Yakimenko, Nucl. Instrum. Methods Phys. Res., Sect. A 608, S18 (2009).

[5] D. J. Gibson et al., Phys. Rev. ST Accel. Beams 13, 070703 (2010).

[6] K. Dobashi et al., Jpn. J. Appl. Phys. 44, 1999 (2005).

[7] T. Omori et al., Phys. Rev. Lett. 96, 114801 (2006).

[8] V. N. Litvinenko, I. Ben-Zvi, D. Kayran, I. Pogorelsky, E. Pozdeyev, T. Roser, and V. Yakimenko, IEEE Trans. Plasma Sci. 36, 1799 (2008).

[9] P. Oliva, M. Carpinelli, B. Golosio, P. Delogu, M. Endrizzi, J. Park, I. Pogorelsky, V. Yakimenko, O. Williams, and J. Rosenzweig, Appl. Phys. Lett. 97, 134104 (2010).

[10] F. H. O'Shea et al. (to be published).

[11] E. S. Sarachik and G. T. Schappert, Phys. Rev. D 1, 2738 (1970).

[12] E. Esarey, S. K. Ride, and P. Sprangle, Phys. Rev. E 48, 3003 (1993).

[13] G. A. Krafft, A. Doyuran, and J. B. Rosenzweig, Phys. Rev. E 72, 056502 (2005).

[14] M. Babzien et al., Phys. Rev. Lett. 96, 054802 (2006).

[15] D. Haberberger, S. Tochitsky, and C. Joshi, Opt. Express 18, 17865 (2010).

[16] M. Schmitt and C. Elliott, IEEE J. Quantum Electron. 23, 1552 (1987).

[17] D. Iracane and P. Bamas, Phys. Rev. Lett. 67, 3086 (1991).

[18] G. Dattoli and G. Voykov, Phys. Rev. E 48, 3030 (1993).

[19] G. Dattoli, V. V. Mikhailin, P. L. Ottaviani, and K. V. Zhukovsky, J. Appl. Phys. 100, 084507 (2006).

[20] H. Motz, J. Appl. Phys. 22, 527 (1951).

[21] L. D. Landau and E. M. Lifshitz, The Classical Theory of Fields (Pergamon Press Ltd., New York, 1951).

[22] J.D. Jackson, Classical Electrodynamics (Wiley, New York, 1962). 